\title{
Formation flying as an innovative air transportation system for long-haul commercial flight: A focus on operational feasibility and potential gain
}

\author{
L.E. Herinckx ${ }^{1}$ \\ Faculty of Aerospace Engineering, Delft University of Technology, the Netherlands \\ Co-authors ${ }^{2}$ \\ T.L.M. Gutleb, R. van Nunen, E. Van Rompuy, D.A. Bos, H.P.A. Dijkers, J. de Wit, H. Radfar, S.E. Sahin \\ Faculty of Aerospace Engineering, Delft University of Technology, the Netherlands \\ and \\ Dr. W.W.A. Beelaerts van Blokland ${ }^{3}$ \\ Faculty of Aerospace Engineering, Delft University of Technology, the Netherlands
}

\begin{abstract}
Formation flying is introduced as a new and innovative air transportation system for long-haul commercial flight. With this paper the operational feasibility of formation flying is addressed, both from a market demand and economic, as well as an air traffic control perspective. Preliminary results of a case study indicate that operating a newly designed aircraft that is optimized for formation flying could cut the fuel consumption over long-haul flights in half compared to current state-of-the-art aircraft. Therefore, this air transportation system proves to be a possible solution to the impending regulations and fines regarding the reduction of $\mathrm{CO}_{2}$ emissions in aviation.
\end{abstract}

\section{Introduction}

$\mathrm{T}$ HE aviation industry is confronted with more and more stringent regulations on noise and emissions. The Lisbon 2020 Vision $^{1}$ introduced by the European Union states that the carbon dioxide footprint of aviation should decrease by fifty percent by the year 2050 compared to 2005 . This goal is to be reached by aiming for an initial increase in fuel efficiency of 1.5 percent per year until the year 2020. In addition, the price of crude oil has tripled since the year $2000^{2}$, which has put an enormous pressure on the economic performance of airlines. Furthermore, the industry is expected to keep growing during the next few decades, yet it is currently unclear whether there will be sufficient capacity from an air traffic control and airport perspective. There is a distinct need for an innovative air transportation system that results in significant reductions in emissions and lower operating costs compared to current aircraft, while creating a higher capacity in the skies and minimizing the additional strain that is put on air traffic control.

The results of the research presented in this paper prove that flying in formation over long-haul distances with an aircraft that is optimized for such flights has the potential to revolutionize commercial air transport by the year 2030 by overcoming many of the challenges that lie ahead.

First, the concept of formation flying, its founding principles and the requirements it imposes on aircraft are explained. Secondly, the feasibility of formation flying from a market point of view is considered. From this research, potential markets and suitable flying routes are identified. An indication of what can be gained with

\footnotetext{
${ }^{1}$ Graduate student, Faculty of Aerospace Engineering, Delft University of Technology, Kluyverweg 1, 2629HS Delft, the Netherlands, AIAA Student Member.

${ }^{2}$ Graduate students, Faculty of Aerospace Engineering, Delft University of Technology, Kluyverweg 1, 2629HS Delft, the Netherlands.

${ }^{3}$ Lecturer, Department of Air Transport Operations, Faculty of Aerospace Engineering, Delft University of Technology, Kluyverweg 1, 2629HS Delft, the Netherlands, AIAA Senior Member.
}

1

American Institute of Aeronautics and Astronautics 
formation flying is given in a preliminary case study, which then leads to conclusions and recommendations on the subject.

\section{Formation Flying}

Both Lissaman ${ }^{3}$ and Hummel ${ }^{4,5}$ have stated that birds can increase their range significantly by flying in formation. Theoretically speaking, this phenomenon could be translated to aircraft as well.

\section{A. Fundamental aerodynamic principles}

Aircraft can benefit from flying in formation by positioning their wings in the upwash region of the trailing wake vortices of a preceding aircraft. As such, the wing in the vortex experiences a decrease in drag due to locally tilted lift vector. Bos et al. ${ }^{6}$ give a detailed explanation of this drag reduction. Similarly to flocks of birds, flying in the upwash region of the vortex results in a reduction in the overall drag of the aircraft.

Unfortunately, there are also some unwanted side effects to flying in a vortex as discussed by Bos et al. ${ }^{6}$. If only one wing flies in the upwash of a vortex a substantial roll moment about the longitudinal axis of the aircraft is induced due to the asymmetric lift. The accompanying drag reduction on one wing induces a yaw moment. Both of these side effects need to be counteracted. The side effects can be compensated with a specially equipped aircraft, such as the Formation Flyer presented by Dijkers and van Nunen ${ }^{7}$.

\section{B. Basic operational setup}

In practice, formation flying is executed by creating a sort of hub in the sky. Aircraft that are expected to more or less be flying along the same route for the majority of their flight can meet up and fly in formation. Each aircraft takes off from its departure airport and flies solo towards a rendezvous point or hub. At this rendezvous point the formation is put together and from here on the aircraft fly together as one group along the trunk route.

To minimize the total fuel consumption of the group and to ensure safety by building in redundancy the aircraft switch positions while flying along the trunk route. As such, each aircraft is in the lead for part of the formation flight. If there would only be one leader this aircraft would need to carry substantially more fuel than the followers, as the leader does not benefit from the formation. In case of an emergency with the leading aircraft the followers would not have sufficient fuel to complete their flight. For this reason each aircraft should be capable of flying in the lead for part of the flight and position switches play an imperative role in formation flight.

When the formation is approaching the destination airports of the individual flights, aircraft can leave the formation one by one. After break-off an aircraft can either continue on for a partial solo flight or it can commence its descent into its destination airport.

\section{Formation geometry and separation}

While birds opt for an inverted-V-shaped formation this is not the most ideal geometry for aircraft. As can be seen in Fig. 1 this geometry is in fact impossible for aircraft because it is not safe for the two aircraft directly behind the leader to fly with a lateral separation of less than one wingspan. In addition, flying in the downwash area of a vortex should be avoided in all cases. Not only does the downwash have a counterproductive effect on the aircraft, it is also not safe to fly in.

Aside from the inverted-V shape many more possible formation geometries such as a simple line, a regular Vshape and an X-shape were considered. However, only the so-called Echelon form depicted in Fig. 2 eliminates the lateral separation issue, while remaining clear of the downwash area of the vortex.

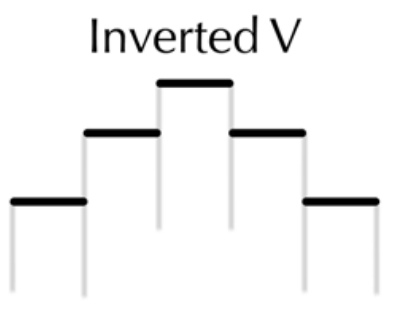

Figure 1. Inverted V formation geometry

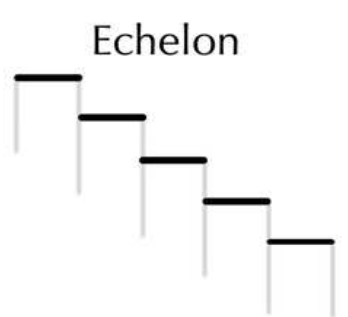

Figure 2. Echelon formation geometry 
Based on the work of Rossow and James ${ }^{8}$, and that of Ginevski and Zhelannikov ${ }^{9}$, both concerning the stages of vortex decay, the ideal separation distance between aircraft in streamwise direction is determined as ten wingspans or $500 \mathrm{~m}(1640 \mathrm{ft})$ in the case of the Formation Flyer, whereas the vertical separation due to vortex decay is around $15 \mathrm{~m}$ (50ft). The lateral separation is dictated by the location of the vortex of the preceding aircraft. Compared to current regulations, which impose a minimum lateral separation of five nautical miles (9260m) and a minimum vertical separation of one thousand feet $(300 \mathrm{~m})$ in uncontrolled airspace, these decreased separation distances would have an enormous impact on the capacity of the airspace.

From an aerodynamic point of view the drag reduction increases as more aircraft are added to the formation. Fig. 3 indicates that this reduction stagnates around the asymptotic value of eighteen percent.

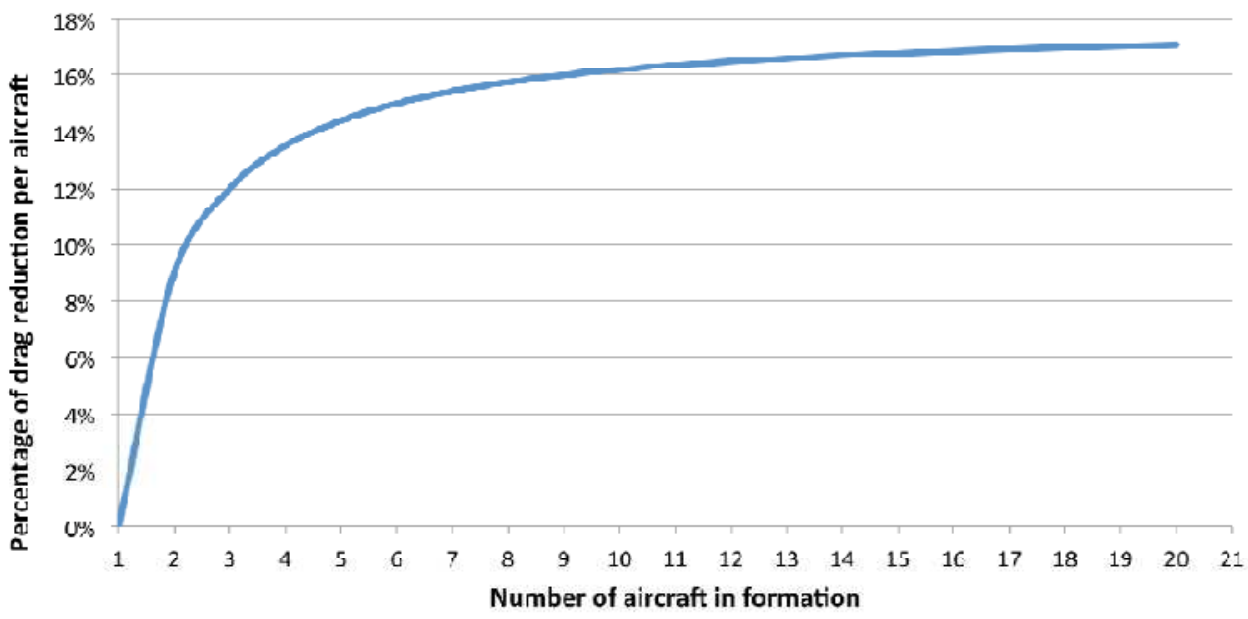

Figure 3. The effect of the number of aircraft in a formation on the drag reduction per aircraft ${ }^{6}$

Although drag reductions are achieved with as little as two aircraft in formation, the benefits are maximal with four aircraft or more. For safety reasons it is recommended that no more than around ten aircraft fly in one formation, as this would mean that one formation takes up a vertical block of five hundred feet of airspace, or five flight levels. In lateral direction the formation would occupy ten wingspans, or around 500m (1640ft) in the case of the Formation Flyer.

\section{Market analysis}

\section{A. Air Traffic Forecast for 2029}

In order to implement formation flying there should be sufficient traffic along the same routes, so that aircraft can easily be put together. The concept can only be successful if the additional distance that aircraft have to fly in formation compared to direct solo flight, the so-called distance penalty, is kept to a bare minimum. For this reason a thorough market analysis consisting of flight routes, as well as traffic density along these routes is done.

Boeing $^{10}$ foresees an overall increase in demand for air transportation according to the graphical representation of forecasted revenue passenger kilometers (RPK) for major intercontinental connections in the year 2029 in Fig. 4. 


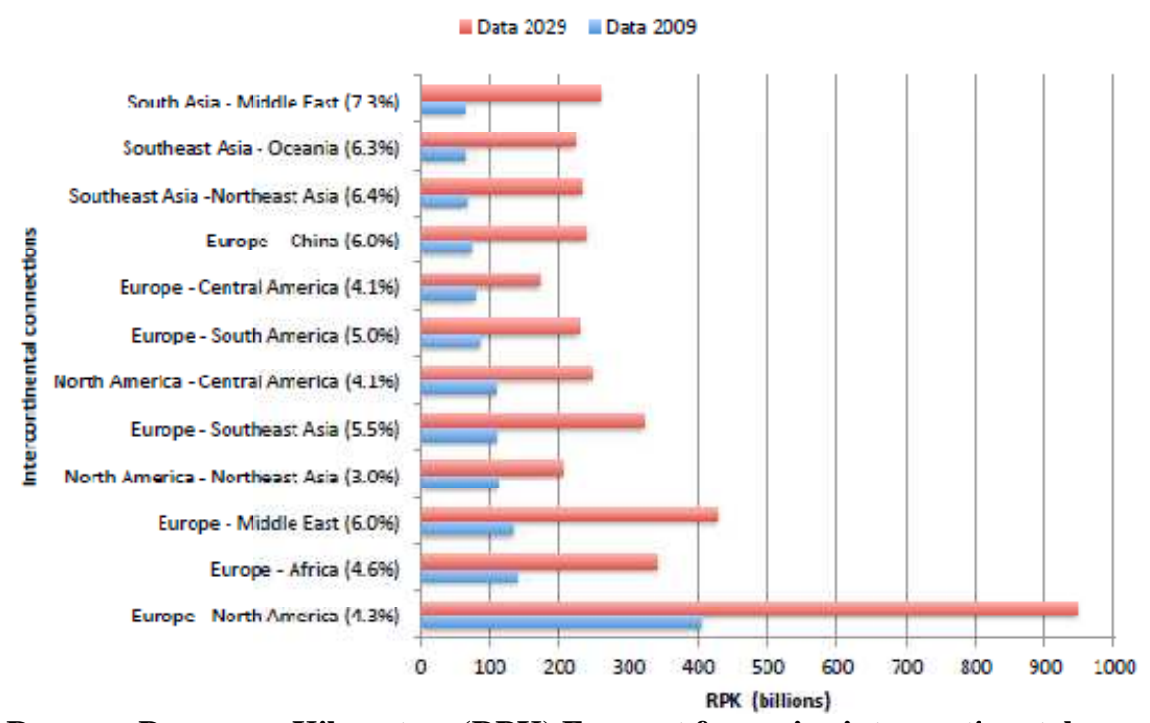

Figure 4. Revenue Passenger Kilometers (RPK) Forecast for major intercontinental connections in 2029 compared to $2009^{10}$

Although there are several connections with a higher average growth rate, the Europe - North America connection is still expected to be the largest long-haul market in 2029. As a starting point, the feasibility of formation flying on this route is considered.

\section{B. Focus on North Atlantic market}

Every day a set of twelve tracks, six Eastbound and six Westbound - the so-called North Atlantic Tracks (NAT), is defined across the North Atlantic based on weather conditions such as jet streams. These tracks, of which an example is given in Fig. 5, form a type of multiple lane highway in the sky. They are to be followed by all aircraft flying along this route.

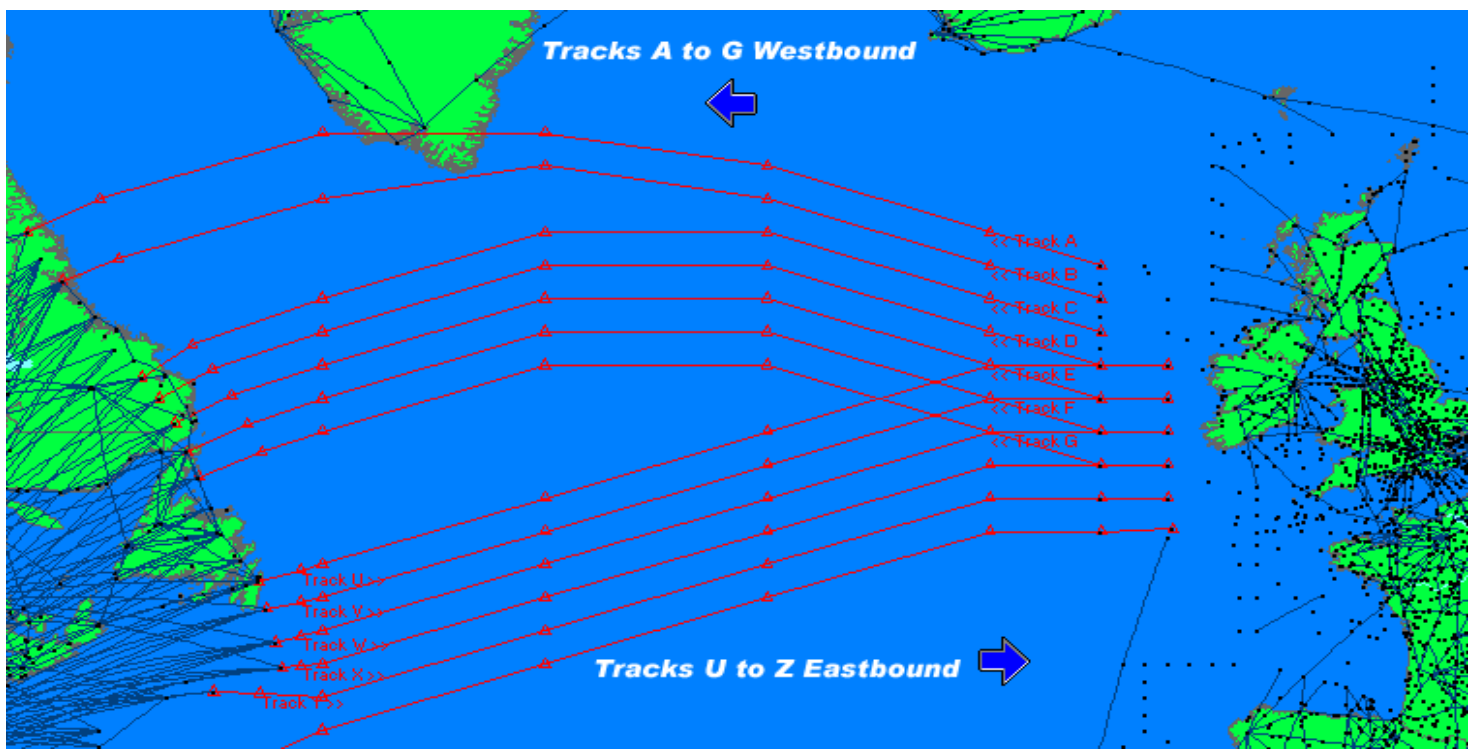

Figure 5. Westbound and Eastbound North Atlantic Tracks (NAT) 
Due to regulations and safety measures a minimum separation of five nautical miles $(9260 \mathrm{~m})$ in horizontal direction and thousand feet $(300 \mathrm{~m})$ in vertical direction must be maintained in uncontrolled airspace. This limits the capacity of the NAT. Nevertheless, there are actually many aircraft more or less in the same place at the same time, which is illustrated in Fig. $6^{11}$ depicting two aircraft on the same track but on different flight levels.

To get a good idea of the traffic density across the North Atlantic, flights between four major European airports (Amsterdam, Frankfurt, London and Paris) and seventeen cities along the East coast of North America were sorted according to their departure time. These European airports account for almost seventy-five percent of the traffic across the North Atlantic.

Figure 7 illustrates that up to thirty aircraft per hour fly Westbound along the NAT every single day. During these peak hours composing formations should be feasible. At quieter times with only two aircraft per hour, it may not be so beneficial to fly in formation, depending on the circumstances.

Given the fact that Boeing ${ }^{10}$ expects the demand for travel between Europe and North America to more than double by the year 2029, as presented in Fig. 4 , it is safe to say that there is a sufficient traffic flow to be able to efficiently compose formations.

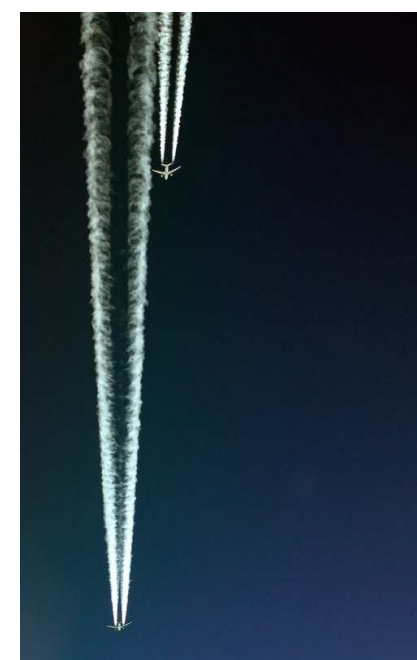

Figure 6. Two aircraft on different flight levels along the same NAT ${ }^{11}$ (used with permission)

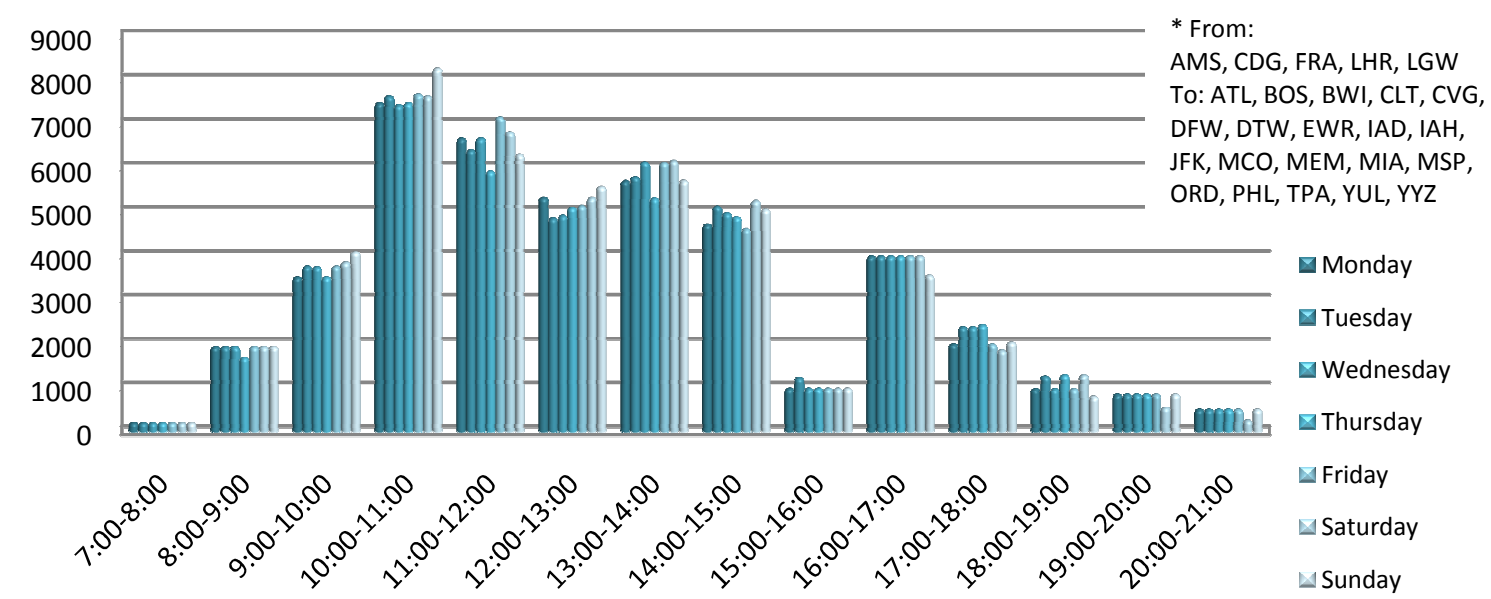

Figure 7. Westbound aircraft density along the NAT* throughout the day from November 22-29, 2010

In order to minimize the distance penalties for all flights considered, while aligning as many major airports across North America as possible, three separate tracks were defined as shown in Fig. 8. The East Coast takes up the vast majority of the flights towards North America. A further distinction can be made between a Southern track (1) and a Northern track (2). Track 1 attracts about two thirds of the East Coast traffic, whereas Track 2 accommodates one third. From here on out track one was chosen for a more detailed case study. 


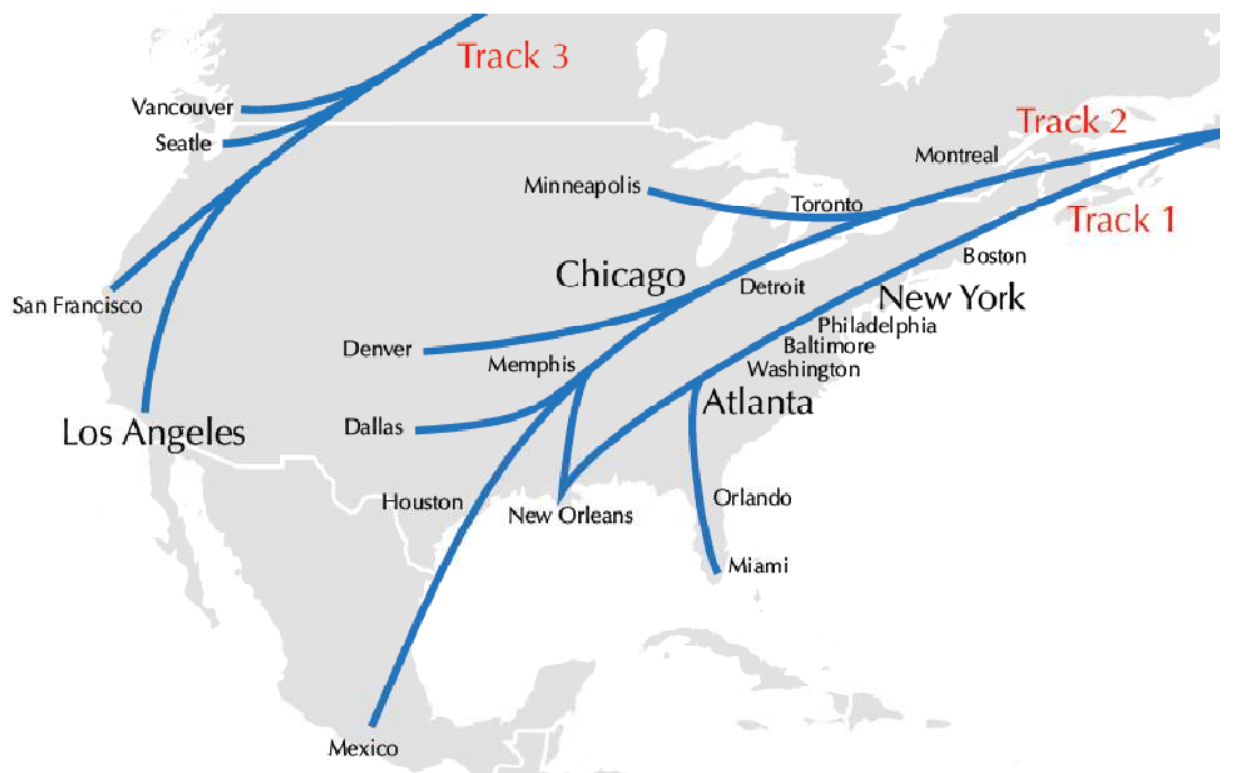

Figure 8. Formation flying tracks between Europe and North America

\section{Global implementation}

Further research on the demand for air traffic and the frequency of flight movements between city pairs lead to the conclusion that formation flying can be implemented as a global concept. With carefully designed tracks such as those presented in Fig. 9 most major cities in the world can be reached with a distance penalty below five percent. There are currently sufficient flight movements along these tracks to make formation flying possible from an operational point of view. As the flight frequency is only expected to increase, insufficient aircraft flow along a route should not be an issue.

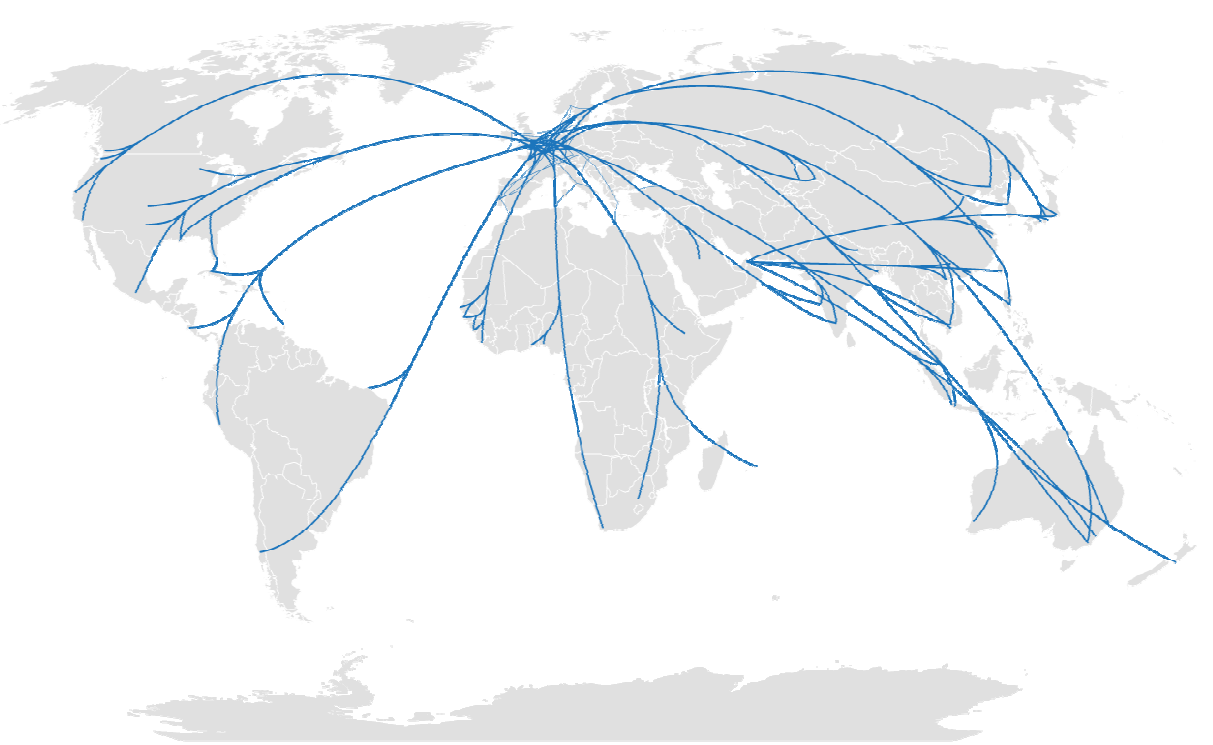

Figure 9. Formation flying tracks from Europe to all major cities in the world

6

American Institute of Aeronautics and Astronautics 


\section{Case study}

In order to get an initial estimate of the benefits of formation flying in practice a case study was done. This analysis also highlighted any vulnerabilities which may be encountered when executing the concept.

\section{A. Setup}

A case study was set up with seven flights taken from flight schedules in November 2010. The flights noted in Table 1 were chosen such that a rendezvous point could easily be reached by all aircraft around the same time. Furthermore, a variety of destinations is included to demonstrate that formation flying can be widely implemented and is not only relevant for destinations which are very close to each other.

During the formation flight the group of aircraft is expected to be fully autonomous. Only one or two aircraft stay in touch with air traffic control. The information is then shared with the whole group. In addition to increasing the capacity of the airspace, the workload of the air traffic control services is kept within moderation, even when more aircraft are flying than is currently the case.

Table 1. Flight schedule for case study

\begin{tabular}{|c|ccc|}
\hline Departure Airport & Arrival Airport & Flight Number & Departure Time (CET) \\
\hline Frankfurt (FRA) & Washington (IAD) & LH418 & $13: 00$ \\
\hline Amsterdam (AMS) & Newark (EWR) & CO71 & $13: 20$ \\
\hline Paris (CDG) & Boston (BOS) & AF332 & $13: 30$ \\
\hline Paris (CDG) & New York (JFK) & AF6 & $13: 30$ \\
\hline Paris (CDG) & Atlanta (ATL) & DL21 & $13: 30$ \\
\hline Paris (CDG) & Newark (EWR) & AF18 & $13: 40$ \\
\hline London (LGW) & Orlando (MCO) & VS15 & $14: 00$ \\
\hline
\end{tabular}

For all but one of these flights the distance penalty is below one percent, as is calculated in Table 2 .

Table 2. Calculation of distance penalties

\begin{tabular}{|c|c|c|c|c|}
\hline Flight & $\begin{array}{c}\text { Total distance with } \\
\text { formation flying [nm] }\end{array}$ & $\begin{array}{c}\text { Total Distance Point- } \\
\text { to-Point [nm] }\end{array}$ & $\begin{array}{c}\text { Penalty } \\
{[\mathbf{n m}]}\end{array}$ & $\begin{array}{c}\text { Distance Penalty } \\
{[\%]}\end{array}$ \\
\hline FRA-IAD & 3,553 & 3,546 & 7 & 0.2 \\
\hline AMS-EWR & 3,198 & 3,177 & 21 & 0.7 \\
\hline CDG-BOS & 3,005 & 2,997 & 8 & 0.3 \\
\hline CDG-JFK & 3,170 & 3,158 & 12 & 0.4 \\
\hline CDG-ATL & 3,846 & 3,819 & 27 & 0.7 \\
\hline CDG-EWR & 3,185 & 3,171 & 14 & 0.4 \\
\hline LGW-MCO & 3,904 & 3,782 & 122 & $\mathbf{3 . 2}$ \\
\hline
\end{tabular}

\section{B. The Formation Flyer}

To demonstrate the full potential of this air transportation system all flights are performed with the Formation Flyer concept aircraft by Dijkers and van Nunen ${ }^{7}$. This aircraft, shown in Fig. 10, was designed for long-haul commercial flight and has a maximum capacity and range similar to the Boeing 787-8. The Formation Flyer is equipped with an integrated Global Navigation Satellite System (GNSS) and Inertial Navigation System (INS) to ensure millimeter-tocentimeter accuracy of relative positioning. This allows

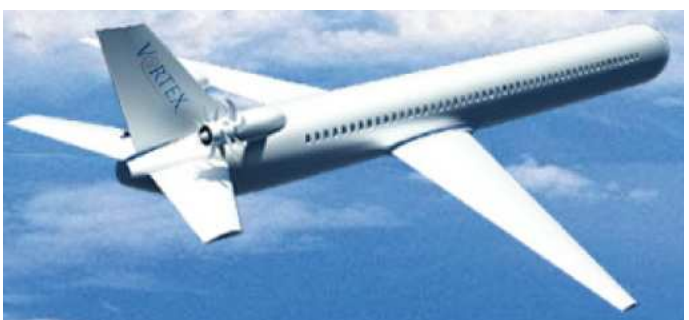

Figure 10. The Formation Flyer concept 
the aircraft to fly within minimal separation distances of each other. Furthermore, there are several LiDAR sensors mounted near the wings and the nose of the aircraft to detect vortices. The side effects of flying in a vortex with only one wing, as is the case in an Echelon formation, are counteracted by a larger-than-average and structurally enhanced tail (yaw moment) and morphing wings (roll moment). The presence of tail-mounted open-rotor jet engines result in a twenty percent decrease in fuel consumption, whereas the noise they produce is shielded towards the ground by the horizontal tail surface. The location of the engines also ensures a clean wing to fully benefit from flying in the upwash of a vortex. The designers of the concept are confident that the technology required to equip this aircraft will be available by the year 2030. All in all, significant fuel savings due to both the highly efficient engines and the benefit of flying in formation lead to a much lighter structure than comparable state-of-the-art aircraft.

\section{Execution of formation flight}

The seven aircraft departing from Amsterdam, Frankfurt, Paris and London form a formation above Shannon, Ireland before continuing as one flock of aircraft across the Atlantic Ocean, as shown in Fig. 11. During this formation flight several switches are performed according to the timeline in Fig. 12 on the next page. During a switch maneuver the leading aircraft turns away from the formation and slightly slows down, until it can take the last position in the Echelon. At this point the LiDAR vortex detection system will have to locate the vortex of the preceding aircraft for the wing to be positioned correctly.

Once the aircraft are nearing Boston the first

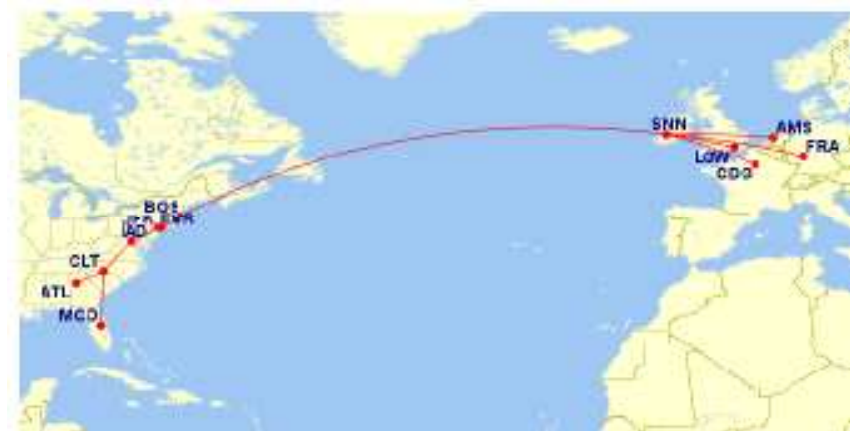

Figure 11. The transatlantic route flown by a formation of seven aircraft aircraft leaves the formation and commences its descent into Boston. The same happens in New York and Washington. After Washington two aircraft remain, which continue to fly in formation until they reach Charlotte, North Carolina. At this point the formation breaks up and both aircraft continue their flight solo to Atlanta and Orlando, respectively.

It is interesting to note that according to the formation flight plan in Fig. 12 the aircraft fly an average of two thirds of their flight in formation. By optimizing the composition of formations the percentage of the flight that is flown in formation can be increased, thereby decreasing the total fuel consumption of the aircraft. In this case this could be achieved by replacing the flights to Atlanta and Orlando with two flights going to the Boston - New York Washington area.

Composing the formations and optimizing this process should be done by a newly established "Formation Flying Authority". This authority could be responsible for defining new regulations concerning formation flight, for instance including break-off procedures in case of emergency. 


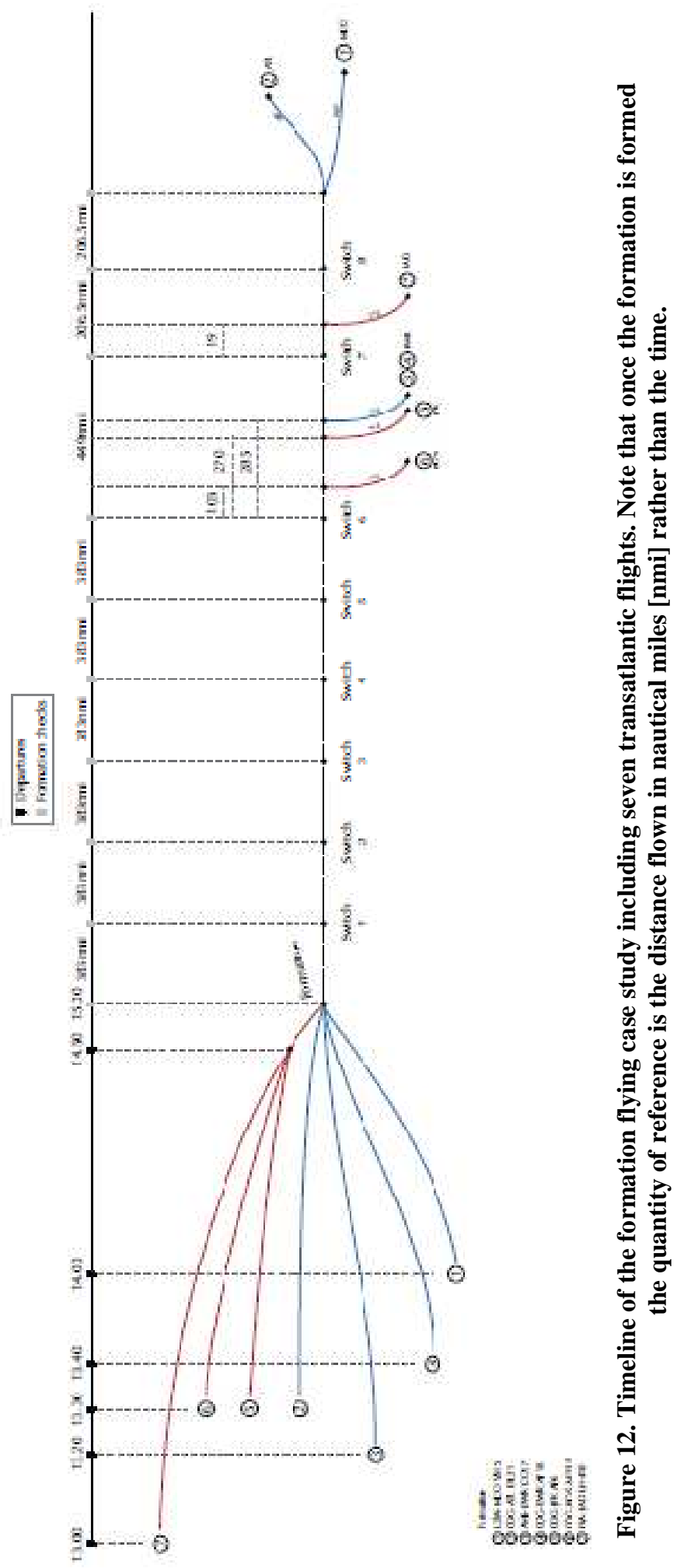




\section{Results: fuel consumption}

In order to quantify the effect formation flying has on the daily operations of an airline the amount of fuel consumed by each of the seven aircraft during each segment of their respective flight is calculated. Using these results the total fuel savings for each aircraft can be found, as well as their effect on the total cost of a flight and the aircraft's emissions.

By dividing a flight into various solo and formation segments, thereby taking into account whether or not the aircraft is benefitting from a vortex, a very good approximation of the total fuel consumption in formation is obtained. As the Formation Flyer has two contributing factors when it comes to fuel savings, namely flying in formation and the open-rotor jet engine, the effect of each of these is treated separately as well as cumulative. In the calculations twenty percent in fuel savings were allotted to the engine, whereas the drag reduction due to flying in formation is accountable for an $18 \%$ decrease in fuel consumption. Additional savings are obtained thanks to lighter aircraft structure that is needed when less fuel needs to be stored.

The baseline for comparison is the fuel consumption of a conventional aircraft on the most optimal trajectory (i.e. the shortest distance) between city pairs. The conventional aircraft used as a reference here is the state-of-the-art Boeing 787-8, which has a very similar range and capacity to the Formation Flyer.

In Table 3 the total fuel consumption for each flight in the formation is presented when a conventional aircraft flies solo and direct, and the Formation Flyer flies in formation. The results indicate that, despite a slight increase in distance flown, the Formation Flyer air transportation system consumes an average of fifty-four percent less fuel than what is considered to be the most fuel efficient long-haul aircraft that is on the market in 2011.

Table 3. Fuel consumption comparison between a conventional aircraft flying point-to-point and the Formation Flyer flying in formation

\begin{tabular}{|c|c|c|c|c|}
\hline Flight & $\begin{array}{c}\text { FF Open-Rotor Jet fuel } \\
\text { used [kg] }\end{array}$ & $\begin{array}{c}\text { Conventional fuel used } \\
{[\mathrm{kg}]}\end{array}$ & $\begin{array}{l}\text { Difference } \\
{[\mathrm{kg}]}\end{array}$ & $\begin{array}{c}\text { FF ORJ Savings vs. } \\
\text { Conventional solo } \\
{[\%]}\end{array}$ \\
\hline VS15 & 27,301 & 59,068 & 31,767 & $53.78 \%$ \\
\hline DL21 & 27,039 & 59,410 & 32,372 & $54.49 \%$ \\
\hline $\mathrm{CO} 71$ & 24,123 & 53,391 & 29,268 & $54.82 \%$ \\
\hline AF18 & 24,056 & 53,334 & 29,278 & $54.89 \%$ \\
\hline AF6 & 23,974 & 53,210 & 29,236 & $54.94 \%$ \\
\hline AF332 & 23,285 & 51,671 & 28,386 & $54.94 \%$ \\
\hline LH418 & 25,707 & 56,872 & 31,166 & $54.80 \%$ \\
\hline Average & 25,070 & 55,280 & 30,210 & $54.65 \%$ \\
\hline
\end{tabular}

\section{E. Results: economic and environmental benefits}

While the Formation Flyer would no doubt be more expensive to purchase than a conventional aircraft, the amount of fuel that can be saved with this concept would have a tremendous impact on the operating cost of the aircraft. In the case study considered each aircraft saves an average of thirty metric tons of fuel, which translates to around USD24,000 per flight using the jet fuel price in January 2011. Based on the assumption that an aircraft performs six hundred flights between Western Europe and the East Coast of the United States in one year, this would result in annual savings of USD14.4 million per aircraft.

An industry whose success is now more than ever dependent on fuel prices could thrive under the economic benefits offered by formation flying. Not to mention, the early compliance with the regulations set to severely limit $\mathrm{CO}_{2}$ emissions from 2050 on. When adopting this concept airlines could reap the benefits of becoming more environmentally friendly while saving millions of dollars. 


\section{Conclusions and recommendations}

This research proves that there is sufficient demand to globally implement formation flying as a new and innovative air transportation system. By using the Formation Flyer aircraft by Dijkers and van Nunen ${ }^{7}$ fuel savings of more than fifty percent are within reach, thereby meeting the Lisbon 2020 Vision requirements for the year 2050 in 2030. Fuel savings of in this order of magnitude would result in massive cost savings for airlines. Furthermore, the work load on air traffic control authorities is reduced, as a flock of two to ten aircraft in a formation can be considered as one block.

To further increase the potential gain of the concept formations could be composed with aircraft with points of origin and points of destination as close together as possible, respectively. As such the portion of the flight that is flown in formation increases. For example, a formation of aircraft with flights from Amsterdam, Brussels, Paris and London to New York would be even more efficient than the formation used in the initial case study.

Some kind of formation flying authority should be established, which receives flight plans from airlines and plans formations. In addition, a clear set of procedures and regulations should be set up to deal with day-to-day operations, as well as any irregularities.

Based on the conclusions presented above it is justified to further investigate formation flying and how it could revolutionize long-haul air travel. Especially on the level of aerodynamics there is a lot of work to be done, as the effects of side wind, aircraft maneuvers and vortex meandering have been omitted in this research.

\section{Acknowledgments}

The authors would like to thank their principal tutor Dr. W.W.A. Beelaerts van Blokland and their coaches Ir. P. Roling, G. Giorgi MSc and Dr. Ir. L.L. Veldhuis for their guidance and support throughout this project.

\section{References}

${ }^{1}$ European Union, Lisbon 2020 Vision, Lisbon, Portugal, 2009.

${ }^{2}$ WTRG Economic, 'Crude oil production of OPEC countries from 1990 to 2009'.

URL: http://www.wtrg.com/oil graphs/PAPRPOP90.gif [cited December 2, 2010]

${ }^{3}$ Lissaman, P. and Shollenberger, C., "Formation Flight of Birds", Science, Vol. 168, 1970, pp. 1003-1005.

${ }^{4}$ Hummel, D., "Aerodynamic Aspects of Formation Flight in Birds", Journal of Theoretical Biology, Vol. 104, No. 3, 1983, pp. 321-347.

${ }^{5}$ Hummel, D., "Formation Flight as an Energy-Saving Mechanism", Journal of Zoology, Vol. 41, No. 3, 1995, pp. 261-278.

${ }^{6}$ Bos, D.A. et al., "Formation Flying Final Report", Faculty of Aerospace Engineering, Delft University of Technology, the Netherlands 2011 (unpublished).

${ }^{7}$ Dijkers, H.P.A, and van Nunen, R., "Integrated Design of a Long-Haul Commercial Aircraft Optimized for Formation Flying”, Faculty of Aerospace Engineering, Delft University of Technology, the Netherlands, 2011 (to be published).

8Rossow, V.J. and James, K.D., “Overview of Wake Vortex Hazards During Cruise”, Journal of Aircraft, Vol. 37, No. 6, 2000, pp. 960-975, NASA Ames Research Center, Moffett Field, California, United States.

\footnotetext{
${ }^{9}$ Ginevski, A.S. and Zhelannikov, A.I., "Vortex Wakes of Aircraft", 2009.

${ }^{10}$ Boeing, “Current Market Outlook 2010-2029”, The Boeing Company, 2010.

${ }^{11}$ Photograph by Terry Bowken, used with permission.
} 